\title{
A Review of Common Methods Used to Exclude Infection in Patients with Lower Urinary Tract Symptoms
}

\author{
Kiren Gill $^{1 *}$ and Govind Dhillon ${ }^{2}$ \\ ${ }^{1}$ Department of Medicine, University College London, UK \\ ${ }^{2}$ Southampton Medical School, Southampton, UK
}

*Corresponding author: Kiren Gill, Department of Medicine, Centre for Nephrology, University College London, London, UK

Submission: 監 October 04, 2017; Published: $\frac{1-1-1}{10}$ November 03, 2017

\begin{abstract}
Lower urinary tract symptoms form a significant burden for many patients. A key step in management of such patients includes the exclusion of urinary tract infection. In patients with classic symptoms of acute infection including frequency and dysuria history alone is sufficient. However in patients with non-dysuric lower urinary tract symptoms, we rely on commonly used investigations to exclude infection. These tests include the midstream urine culture, microscopy of urinary and urinary dipstick tests. Our understanding of urinary infection has advanced, however the tests we use in clinically practice do not exclude urinary tract infection. This review explores the role and inadequacies in the current tests used to exclude urinary infection in patients that present with urinary infection.
\end{abstract}

Abbreviations: LUTS: Lower Urinary Tract Symptoms; UTI: Urinary Tract Infection; PPV: Positive Predictive Value; NPV: Negative Predictive Valve; CSU : Catheter Specimen of Urine

\section{Review}

In patients who present with lower urinary tract symptoms (LUTS) it is important to exclude the presence of bacterial infection which may be responsible for the presenting symptoms. In patients presenting with acute frequency and dysuria, diagnosis is based on history alone. However, in patients presenting without dysuria we rely on investigations to help exclude urinary tract infection (UTI). Internationally and in the UK these include the quantitative cleancatch midstream urine culture, which is used as the gold standard for diagnosis, as recommended by NICE (2015) in the UK. In addition, as recommended by NICE and the International Continence Society (ICS) $(2002,2010)$ microscopy of urine, bedside testing with urine dipsticks to test for leucocyte esterase and nitrites as well as microbiological culture of urine, is used to test for infection.

Published guidelines across Europe, USA and the UK reveal significant discrepancies in the choice of a quantitative threshold used to define significant bacteriuria. The clean-catch, midstream urine (MSU) sample culture in the UK and Europe commonly uses the Kass (1957) criterion of $10^{5}$ colony forming units (cfu) $\mathrm{ml}^{-1}$ of a single species of a known urinary pathogen [1,2]. Kass drew these data from 74 women with acute pyelonephritis and 337 normal controls, a select sample unrepresentative of wider lower urinary tract symptoms (LUTS). Despite its limitations, this criterion has become a ubiquitous reference standard and has been challenged by several groups $[3,4]$. The Associate of Urology (EUA) guidelines for urological infections emphasize that no single threshold can be applied in all clinical situations. The dipstick testing has been validated against the Kass criteria. It is now well documented in the literature that the urinary dipstick tests used for beside testing for nitrite and leucocyte esterase are unreliable [5-8].

The quantitative microbiological bacterial culture remains the reference gold standard in diagnosis of significant bacteria. Currently in the UK the proposed threshold that discriminates normal from pathology is $10^{5} \mathrm{cfu} \mathrm{ml}^{-1}$. This threshold is based on the original work by Kass with a positive culture being defined as greater than $10^{5} \mathrm{cfu} \mathrm{ml}^{-1}$ of a single known urinary pathogen was significant of urinary tract infection in this group of patients. This continues to be used as the threshold that discriminates between normal and 'urinary tract infection' in patients who present with lower urinary tract symptoms, in many centres internationally. There has since been other work in patients presenting with classic symptoms of acute cystitis with sudden onset of pain, frequency and urgency. It was found that a threshold of $10^{5} \mathrm{cfu} \mathrm{ml}^{-1}$ missed nearly $50 \%$ of genuine coliform infections. It was proposed that 
in patients with symptoms of acute cystitis, bacterial growth of greater than $10^{2} \mathrm{cfu} \mathrm{ml}^{-1}$ of a known urinary pathogen was a more appropriate threshold in this group of patients [3,9]. Unfortunately however much of this work has been overlooked and in patients who present with LUTS, many laboratories still define a positive culture as growth of more than $10^{5} \mathrm{cfu} \mathrm{ml}^{-1}$ of a known urinary pathogen.

In clinical medicine, to aid diagnosis we frequently use absolute terms to describe the existence or absence of disease. However nature and biology do not allow such distinct entities and commonly disease manifests across a spectrum [10]. In patients describing symptoms of acute frequency and dysuria the diagnosis of UTI is not challenging and should be based on history alone. However in patients presenting with non-dysuric symptoms we still rely on the use of current investigations to help exclude urinary infection. It is now widely appreciated that in patients with lower urinary tract symptoms, a midstream urine sample that is reported as negative based on the $10^{5} \mathrm{cfu}^{-1} \mathrm{l}^{-1}$ threshold does not exclude significant UTI $[3,8,11-14]$. It is also important to consider that nature works does not present with categories but to consider a continuum.

The work by Kass also only addressed pathogenic coliform organisms, and mixed growth was thought to be likely due to contamination and was dismissed. There is now evidence to support polymicrobial infection in human disease, with work exploring this in the bladder [4]. The culture methods used today also assume dominant pathogenicity from the Enterobacteriaceae species, notably E coli. Because of this assumption, the MSU culture is performed on a selective medium (selective chromogenic medium) for Enterobacteriaceae under aerobic conditions. This does not allow for the growth of anaerobic bacteria and other pathogens, which do not grow well under such culture conditions. Whilst this might be appropriate for diagnosing acute infection, there is no evidence that the same bacteria are implicated in chronic infections or patients presenting with non-dysuric lower urinary tract symptoms such as in the overactive bladder. It is therefore not appropriate to use the current MSU culture for such patients and this is an avenue that warrants further explorations.

Microscopy of urine for the detection of urinary leucocytes is widely used as a surrogate marker of urinary infection. Due to the time, cost and effort of MSU cultures, in some laboratories, microscopy of pyuria acts as a screening tool where only those samples with significant microscopic pyuria are then sent for culture. This technique stems from Dukes work in 1927 which predates Kass' work. His work reviewed midstream urine samples from 300 asymptomatic volunteers and found a mean leucocyte counts of $1.6 \mathrm{wbc} \mu \mathrm{l}^{-1}$ in males and $5.4 \mathrm{wbc} \mu \mathrm{l}^{-1}$ in females with a

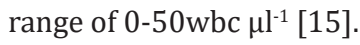

From this, he proposed an arbitrary cut off of $<10 \mathrm{wbc} \mu \mathrm{l}^{-1}$ as the limit for normal pyuria. As a consequence we now use a microscopic pyuria $\geq 10 \mathrm{wbc} \mu^{-1}$ to suggest urinary infection. The work by Dukes is open to similar criticism to Kass' work of spectrum bias. Here a healthy population has been used to define disease in a symptomatic population. In addition the data from Dukes work shows a wide range of pyuria and hence the data would be skewed and not normally distributed. Therefore use of the median would have been a more appropriate measure of central tendency and had that occurred, zero pyuria would have been promoted as the normal state. The samples collected in Dukes' work were also voided samples and without careful sampling may be subject to contamination. Studies comparing catheter sampling do not report any differences in leucocyte excretion between the sexes.

Microscopic pyuria is commonly used to exclude infection and many studies have reviewed its performance against quantitative bacterial culture. Some studies have however found that those with symptoms of acute UTI were culture negative, against a threshold of $\geq 10^{5} \mathrm{cfu} \mathrm{ml}^{-1}$ as diagnostic of infection, nearly half demonstrated leucocyte excretion of $\geq 10 \mathrm{wbc} \mu^{-1}[16,17]$. These data support pyuria excretion $\leq 10 \mathrm{wbc} \mu \mathrm{l}^{-1}$ as a common finding amongst women with acute cystitis symptoms, and questions the microbiological definition of UTI, long before Kass' work was re-evaluated. This history illustrates the error of propositions being accepted as explanatory in the absence of empirical support.

There are limited data evaluating the role of microscopic pyuria as a surrogate marker of infection in patients with non-acute symptoms. In patients with painless LUTS microscopic pyuria has been reported to have a sensitivity of 56\% (95\% CI 46-60\%) and specificity of $72 \%$ (95\% CI $67-76 \%$ ) for midstream urine (MSU) cultures at $\geq 10^{5} \mathrm{cfu} \mathrm{ml}^{-1}$, and $66 \%$ (95\% CI 54-77\%) and $73 \%$ (95\% CI 69-78\%) respectively for catheter specimen of urine (CSU) culture at $\geq 10^{5} \mathrm{cfu} \mathrm{ml}^{-1}$ [18]. In patients presenting with non-acute symptoms Kupelian et al. [19] found the positive predictive value (PPV) and negative predictive valve (NPV) of pyuria as a surrogate marker of UTI to be 0.40 (CI $0.37-0.43$ ) and 0.75 (CI 0.73-0.76) respectively. They found that $40 \%$ of the inflammatory signal was lost by 4 hours after sample collection which is a common delay between patients providing samples and time taken to arrive at laboratories for analysis [19]. Given that microscopy of urine is performed by laboratories so that only those with $\geq 10 \mathrm{wbc} \mu \mathrm{l}^{-1}$ require culture samples may be wrongly dismissed as negative for infection.

Urinary dipsticks are commonly used in both primary and secondary care as a bed side test and in point of care testing when excluding urinary infection. They were introduced as part of 'point of care testing' to aid in diagnosis of UTI and reduce the need for urine microscopy and urine culture and allow for earlier treatment. Modern reagent strips are able to provide information on a variety of physical and biochemical variables. The principle measure of UTI includes the presence of leucocyte esterase and nitrites. Leucocyte esterase is a potential measure of pyuria and the Nitrite test uses the Greiss reaction to detect a nitrate reduction product of some uropathogenic bacteria [20]. The International Consultation on Incontinence guidelines on LUTS strongly recommends the need to exclude UTI and recommends dipstick testing as a screening method [21]. NICE also recommends the use of urinary dipstick for screening patients with lower urinary tract symptoms [22]. However, there are no published data to support dipstick as a valid 
screening tool for patients with non-acute, non-dysuric symptoms. On reviewing the literature there is considerable evidence to question the accuracy of urinary dipsticks [5,6,23,24]. The sensitivity of leucocyte esterase has been suggested to be around $60 \%$ and specificity of around $70 \%$. The sensitivity of nitrite is approximately $18 \%-50 \%$, with reported specificities of $90 \%[5,24]$. However this is using a gold standard as an MSCU culture with a positive threshold as $\geq 10^{5} \mathrm{cfu} \mathrm{ml}^{-1}$.

In assessing anyone who presents with LUTS a mandatory step is the exclusion of UTI, but given the deficiencies in the tests diagnosis may be flawed. We must therefore be aware of this and take into consideration the potential for infection. This warrants further research into how we identify and exclude infection, as well as re-evaluates the pathophysiology of common urological and urogynaecological conditions.

\section{References}

1. Kass EH (1957) Bacteriuria and the diagnosis of infection in the urinary tract. Arch Intern Med 100(5): 709-714.

2. Kass EH (1960) Bacteriuria and pyelonephritis of pregnancy. Arch Intern Med 105: 194-198.

3. Stamm WE, Counts GW, Running KR, Fihn S, Turck M, et al. (1982) Diagnosis of coliform infection in acutely dysuric women. N Engl J Med 307(8): 463-468.

4. Siegman-Igra Y (1994) The significance of urine culture with mixed flora. Curr Opin Nephrol Hypertens 3(6): 656-659.

5. Deville WL, Yzermans JC, van Duijn NP, Bezemer PD, van der Windt DA, et al. (2001) The urine dipstick test useful to rule out infections. A metaanalysis of the accuracy. BMC Urol 4: p. 4.

6. Hurlbut TA, Littenberg B (1991) The diagnostic accuracy of rapid dipstick tests to predict urinary tract infection. Am J Clin Pathol 96(5): 582-588.

7. Williams GJ, Macaskill P, Chan SF, Turner RM, Hodson E, et al. (2010) Absolute and relative accuracy of rapid urine tests for urinary tract infection in children: a meta-analysis. Lancet Infect Dis 10(4): 240-250.

8. Khasriya R, Khan S, Lunawat R, Bishara S, Bignall J, et al. (2010) The Inadequacy of Urinary Dipstick and Microscopy as Surrogate Markers of Urinary Tract Infection in Urological Outpatients With Lower Urinary Tract Symptoms Without Acute Frequency and Dysuria. J Urol 183(5): 1843-1847.

9. Latham RH, Wong ES, Larson A, Coyle M, Stamm WE (1985) Laboratory diagnosis of urinary tract infection in ambulatory women. JAMA 254(23): 3333-3336
10. Richard D (2004) Amphibians-The Salamander's Tale. In: Weidenfield \& Nicholoson (Eds.) The Ancestor's Tale: A Pilgrimage to the Dawn of Life. ( $1^{\text {st }}$ edn), London, UK.

11. Stamm WE (1984) Quantitative urine cultures revisited. European journal of clinical microbiology 3(4): 279-281.

12. Hooton TM, Stamm WE (1997) Diagnosis and treatment of uncomplicated urinary tract infection. Infect Dis Clin North Am 11(3): 551-581.

13. Rosen DA, Hooton TM, Stamm WE, Humphrey PA, Hultgren SJ (2007) Detection of intracellular bacterial communities in human urinary tract infection. PLoS medicine 4(12): e329.

14. Khasriya R, Sathiananthamoorthy S, Ismail S, Kelsey M, Wilson M, et al. (2013) Spectrum of bacterial colonization associated with urothelial cells from patients with chronic lower urinary tract symptoms. J Clin Microbiol Jul 51(7): 2054-2062.

15. Dukes C (1928) Some Observations on Pyuria. Proc R Soc Med 21(7): 1179-1183.

16. Mond NC, Percival A, Williams JD, Brumfitt W (1965) Presentation, diagnosis, and treatment of urinary-tract infections in general practice. Lancet 1(7384): 514-516.

17. Gray LA, Pingelton WB (1956) Pathological lesions of the female urethra. JAMA 162(15): 1361-1365.

18. Khasriya R, Sathiananthamoorthy S, Ismail S, Kelsey M, Wilson M, et al. The spectrum of bacterial colonisation associated with urothelial cells from patients with chronic lower urinary tract symptoms (Epub ahead of print). J Clin Microbiol doi: 10.1128/JCM.03314-12.

19. Kupelian AS, Horsley H, Khasriya R, Amussah RT, Badiani R, et al. (2013) Discrediting microscopic pyuria and leucocyte esterase as diagnostic surrogates for infection in patients with lower urinary tract symptoms: results from a clinical and laboratory evaluation. BJU int 112(2): 231238.

20. McClatchey KD (2001) Clinical laboratory medicine. ( $2^{\text {nd }}$ edn) Lippincott Williams \& Wilkins.

21. Abrams P, Andersson KE, Birder L, Brubaker L, Cardozo L, et al. (2010) Fourth International Consultation on Incontinence Recommendations of the International Scientific Committee: Evaluation and treatment of urinary incontinence, pelvic organ prolapse, and fecal incontinence. Neurourol Urodyn 29(1): 213-240.

22. Jose SS, Sharif ES (2003) New NICE guidelines for UTI management: what will change? Archives of disease in childhood. 93(8): 716-717.

23. Kahlmeter G (2003) Prevalence and antimicrobial susceptibility of pathogens in uncomplicated cystitis in Europe. The ECO.SENS study. Int J Antimicrob Agents 22(Suppl 2): 49-52.

24. St JA, Boyd JC, Lowes AJ, Price CP (2006) The use of urinary dipstick tests to exclude urinary tract infection: a systematic review of the literature. Am J Clin Pathol 126(3): 428-436. 